\title{
MUCORALES (ZYGOMYCOTINA) DA MATA ATLÂNTICA DA RESERVA BIOLÓGICA DO ALTO DA SERRA DE PARANAPIACABA, SANTO ANDRÉ, SP1
}

\author{
Iracema Helena Schoenlein-Crusius ${ }^{2}$ \\ Adauto Ivo Milanez ${ }^{2}$
}

Enviado em 27/0/97. Aceito em 15/8/97.

RESUMO - (Mucorales (Zygomycotina) da Mata Atlântica da Reserva Biológica do Alto da Serra de Paranapiacaba, Santo André, SP). Treze táxons de Mucorales (Zygomycotina), distribuídos num total de 266 registros, foram isolados de folhas de Alchornea triplinervia (Spreng.) Müll. Arg. colocadas em ambientes terrestre e aquático, e de amostras de solo e de água de riacho, coletadas mensalmente, de julho de 1988 a maio de 1990 na Reserva Biológica do Alto da Serra de Paranapiacaba, no município de Santo André, SP. As espécies que apresentaram os maiores números de registro de ocorrência foram Mucor hiemalis Wehmer (78 registros), Mucor circinelloides van Tieghem f. janssenii (Ledner) Schipper (42 registros) e Rhizopus arrhizus Fischer (30 registros). Cinqüenta por cento dos Mucorales encontrados na região são constituídos por espécies que estão sendo citadas pela primeira vez para a Mata Atlântica: Mucor hiemalis Wehmer $\mathrm{f}$. silvaticus (Hagem) Schipper, Rhizopus arrhizus Fischer, Rhizopus oligosporus Fischer, Rhizopus oryzae (Went. \& Prinsen) Geerlings, Zygorhynchus japonicus Vuill. e Zygorhynchns macrocarpus Ling-Young.

Palavras-chave: Mata Atlântica, Zygomycotina, Mucorales, diversidade, Alchornea triplinervia

\begin{abstract}
Mucorales (Zygomycotina) of the Atlantic Rainforest in the "Reserva Biológica do Alto da Serra de Paranapiacaba", Santo André, SP, Brazil). Thirteen taxa of Mucorales (Zygomycotina), distributed in 266 occurrences were isolated from leaves of Alchornea triplinervia (Spreng.) Müll. Arg. disposed in the terrestrial and aquatic environment, and of soil and stream water samples, monthly taken from July of 1988 to May of 1990, in the "Reserva Biológica do Alto da Serra de Paranapiacaba", in the municipality of Santo André, São Paulo State, Brazil. Mucor hiemalis Wehmer (78 records), Mucor circinelloides van Tieghem f. janssenii (Ledner) Schipper (42 records) and Rhizopus arrhizus Fischer (30 records) were the most frequent species. Fifty percent of the Mucorales found are formed by species that are mentioned for the first time in the Atlantic Rainforest: Mucor hiemalis Wehmer f. silvaticus (Hagem) Schipper, Rhizopus arrhizus
\end{abstract}

\footnotetext{
' Parte da tese de Doutorado da primeira autora

${ }^{2}$ Instituto de Botânica, C. P. 4005, CEP 01061-970, São Paulo, SP, Brasil
} 
Fischer, Rhizopus oligosporus Fischer, Rhizopus oryzae (Went. \& Prinsen) Geerlings, Zygorhynchus japonicus Vuill. and Zygorhynchus macrocarpus Ling-Young.

Key-words: Atlantic Rainforest, Zygomycotina, Mucorales, diversity, Alchornea triplinervia

\section{Introdução}

Os representantes de Mucorales (Zygomycotina) caracterizam-se pela reprodução assexuada efetuada pela formação de esporângios ou esporangíolos uni ou multiesporados (merosporos, esporangiosporos, artrosporos e clamidosporos), reprodução sexuada por zigosporos e pelos hábitos sapróbios cosmopolitas (raramente micoparasitas), parasitas facultativos de plantas, animais e do homem (Hesseltine \& Ellis 1973; Hawksworth et al., 1995)

As espécies de Mucorales geralmente são sapróbias, decompondo resíduos vegetais ou animais, bem como excrementos, assumindo o papel de coprófilos (Hesseltine \& Ellis 1973; Lacaz et al. 1970). A bagagem enzimática desses fungos permite-lhes decompor açúcares de estrutura molecular mais simples (Hesseltine \& Ellis 1973), geralmente fazendo-se presente nos estádios iniciais ou finais da decomposição dos substratos orgânicos (Maia 1983; Schoenlein-Crusius \& Tauk 1991), contribuindo significativamente para a ciclagem de nutrientes.

Estudos sobre a taxonomia e sistemática de Mucorales no Estado de São Paulo ainda são escassos, encontrando-se na literatura artigos sobre a diversidade de espécies de Mucor (Trufem 1981a), Absidia, Gongronella, Rhizopus (Trufem 1981b) Circinella e Cunninghamella (Trufem 1981c) e Pilobolus (Viriato \& Trufem 1985), algumas isoladas de amostras de solo coletadas na Mata Atlântica dos municípios de Juquitiba e Registro. Posteriormente foram estudadas espécies coprófilas, isoladas de fezes de diversos animais (Trufem 1984; Trufem \& Viriato 1985; Viriato \& Trufem 1985; Schoenlein-Crusius et al. 1996; Viriato 1996). Alguns representantes de Mucorales também se fizeram presentes nos estudos sobre os fungos da Reserva Ecológica da Juréia-Itatins (Attili 1994; Garlipp 1995).

Segundo Antunes et al. (1993) a ocorrência de espécies de Mucorales no solo foi estimulada por uma queimada acidental na Mata Atlântica de Paranapiacaba.

Representantes de Mucorales também foram isolados de folhas em decomposição de Ficus microcarpa L.f. submersas em um lago artificial no Parque Estadual das Fontes do Ipiranga (Schoenlein-Crusius \& Milanez 1989) e de folhas de Quercus robur L. submersas em um lago no município de Itapecerica da Serra (Schoenlein-Crusius et al. 1990), ampliando o conhecimento sobre a distribuição desses fungos nos ambientes aquáticos.

De acordo com a literatura acima mencionada, pouco se conhece sobre a diversidade dos representantes de Mucorales na Mata Atlântica no Estado de São Paulo, havendo necessidade de estudos mais detalhados sobre esses aspectos.

Foi realizado um estudo comparativo da sucessão fúngica em folhas de Alchornea triplinervia (Spreng.) M. Arg. colocadas sobre o solo e submersas em um dos riachos na Reserva Biológica do Alto da Serra de Paranapiacaba, verificando-se 
inclusive a influência das populações fungicas nativas ( solo e água) sobre a decomposição dos substratos (Schoenlein-Crusius 1993). A apresentação e discussão das ocorrências e distribuição dos treze táxons de Mucorales obtidos no estudo acima mencionado constituem os objetivos do presente artigo.

\section{Material e métodos}

O presente estudo foi conduzido em áreas delimitadas dentro da Mata Atlântica na Reserva Biológica de Paranapiacaba, SP, que se encontra sob a jurisdição do Instituto de Botânica em São Paulo e se localiza no município de Santo André, São Paulo. As características do solo, do clima e da vegetação foram detalhadamente descritas em Schoenlein-Crusius (1993).

Os fungos foram obtidos de amostras de solo, água e folhas em decomposição coletadas do ambiente aquático e terrestre. Para isso foram escolhidas cinco áreas de $50 \mathrm{~m}^{2}$ próximas a um riacho, ao longo do qual foram estabelecidas cinco estações de coleta ( $50 \mathrm{~m}$ de distância entre as mesmas).

Folhas recém-caídas de $A$. triplinervia, uma das espécies mais abundantes na reserva, foram confinadas em 400 sacos de tela de náilon $(20 \times 20 \mathrm{~cm})$ e malha com $1 \mathrm{~mm}$ de diâmetro. Cada saco continha aproximadamente $20 \mathrm{~g}$ (equivalente a 30-40 unidades) de folhas. Metade dos sacos foi distribuída nos cinco pontos de coleta ao longo do riacho e o restante distribuída sobre o solo das áreas demarcadas. Mensalmente, de julho de 1988 a maio de 1990 , foram recolhidos 15 sacos de cada ambiente, juntamente com cinco amostras compostas de solo (aproximadamente $300 \mathrm{~g}$ ) e cinco amostras compostas de água do riacho (aproximadamente $250 \mathrm{ml}$ coletados a $20 \mathrm{~cm}$ de profundidade de cada ponto).

As folhas coletadas foram submetidas à técnica de lavagem de discos de folhas (Pugh et al. 1972), que consiste em lavar discos com diâmetro de 5mm (500 unidades no presente estudo), com água destilada esterilizada $(50 \mathrm{ml})$ sucessivamente por 30 vezes, com a finalidade de eliminar propágulos aderidos à superficie foliar. Os discos foram dispostos de modo eqüidistante em 20 placas de Petri (cada uma com 5 discos), sobre os seguintes meios de cultura: batata-dextrose-ágar, amido-ágar, pectina-ágar (Difco Manual 1972) e celulose-ágar (Eggins \& Pugh 1962), perfazendo 80 placas de Petri contendo discos de folhas submersas e 80 placas contendo discos de folhas coletadas no ambiente terrestre. Alíquotas de $1 \mathrm{ml}$ das amostras de água do riacho foram distribuídas em 40 placas de Petri, cada 10 unidades contendo os mesmos meios de cultura utilizados para o isolamento dos fungos das folhas.

Para isolar os fungos das cinco amostras de solo foi utilizado o método da placa-de-solo (Warcup 1950), modificado por uma diluição (1:100) das alíquotas. Foram semeados $1 \mathrm{ml}$ da diluição em placas de Petri (40 placas) utilizando-se os mesmos meios de cultura empregados para as folhas.

Após sete dias em placas de Petri à temperatura ambiente $\left(20\right.$ a $\left.25^{\circ} \mathrm{C}\right)$, as colônias de Mucorales foram transferidas para o meio de cultura "Synthetic Mucor Agar-SMA” (Schipper 1973) para serem identificadas, sempre que possível, a nível de 
espécie, com auxílio de literatura especializada (Zycha et al. 1969; Schipper 1973; 1976; Trufem, 1978; Domsch et al. 1980).

As culturas identificadas foram preservadas pela técnica de imersão de blocos de ágar em água destilada esterilizada e/ou liofilização. A descrição detalhada dos métodos de preservação e a numeração das culturas estão apresentadas em Schoenlein-Crusius et al. (1994).

\section{Resultados e discussão}

Foram isolados treze táxons referentes a 266 registros de fungos pertencentes à ordem Mucorales (Tab. 1).

Tabela 1. Número de registros e das espécies de Mucorales (Zygomycotina) isolados de amostras de água (A), solo (S) e de folhas de Alchornea triplinervia submersas (FA) e colocadas no ambiente terrestre (F S) na Reserva Biológica do Alto da Serra de Paranapiacaba, Santo André, SP, de julho de 1988 a maio de 1990

\begin{tabular}{lrrrrr}
\hline Táxons & A & S & FA & FS & Total \\
\hline Absidia cylindrospora Hagem & 0 & 12 & 0 & 7 & 19 \\
M. circinelloides van Tieghem f. janssenii (Lendner) Schipper & 3 & 9 & 11 & 19 & 42 \\
M. genevensis Wehmer & 0 & 0 & 0 & 8 & 8 \\
M. hiemalis Wehmer f. hiemalis Wehmer & 20 & 23 & 16 & 19 & 78 \\
M. hiemalis Wehmer f. silvaticus (Hagem) Schipper & 0 & 0 & 7 & 12 & 19 \\
M. racemosus Fres. f sphaerosporus (Hagem) Schipper & 0 & 0 & 0 & 7 & 7 \\
Rhizopus arrhizus Fischer & 2 & 17 & 6 & 5 & 30 \\
R. oligosporus Fischer & 0 & 0 & 0 & 5 & 5 \\
R. oryzae (Went. \& Prinsen) Geerlings & 0 & 12 & 5 & 4 & 21 \\
Zygorhynchus japonicus Vuill. & 0 & 0 & 0 & 6 & 6 \\
Z. macrocarpus Ling-Young & 0 & 6 & 0 & 4 & 10 \\
Z. moelleri Vuill. & 1 & 11 & 3 & 6 & 21 \\
\hline Totais & 26 & 90 & 48 & 102 & 266 \\
\hline
\end{tabular}

No presente estudo os representantes de Mucorales desenvolveram-se abundantemente, produzindo elevada quantidade de esporos, principalmente em meio de amido-ágar, seguido por batata-dextrose-ágar e pectina-ágar.

Nas folhas de Alchornea triplinervia colocadas no ambiente terrestre verificou-se o maior número de ocorrências de Mucorales, com 102 registros totais. Por outro lado, no ambiente aquático foram verificadas 48 ocorrências e nas amostras de água do riacho somente 26 registros. No solo foram registradas 90 ocorrências desses fungos, indicando a afinidade dos mesmos pelo ambiente terrestre

No presente estudo, o gênero Mucor apresentou-se mais frequente (154 registros), sendo representado por quatro espécies e duas variedades, seguido por Rhizopus (56 registros),Zygorynchus ( 37 registros) que se fizeram representar por três espécies de cada gênero e Absidia, com uma única espécie e com 19 registros (Tab. 1).

Absidia cylindrospora ocorreu somente no ambiente terrestre, tendo sido isolada 12 vezes do solo e sete das folhas de Alchornea triplinervia. Esta espécie tem sido 
freqüientemente encontrada no solo de Paranapiacaba (Antunes et al. 1993) e no solo do Parque Estadual das Fontes do Ipiranga (Schoenlein-Crusius et al. 1996; Viriato 1996),

Mucor hiemalis e $M$. circinelloides, com suas diversas formas, constituem táxons bastante freqüentes no ambiente terrestre (Domsch et al. 1980), sendo que também foram encontrados no solo da Estação Ecológica de Juréia-Itatins (Garlipp 1995) e no Parque Estadual das Fontes do Ipiranga (Viriato 1996).

Nas folhas submersas de Ficus microcarpa (Schoenlein-Crusius \& Milanez 1989) e de Quercus robur (Schoenlein-Crusius et al. 1990) foram isoladas apenas Mucor hiemalis e $M$. circinelloides. Antunes et al. (1993) também mencionaram estas espécies para o solo de Paranapiacaba, além de $M$. racemosus. Com exceção de $M$. hiemalis f. silvaticus, aqui relatado pela primeira vez na Mata Atlântica no Estado de São Paulo, todas as demais espécies de Mucorforam anteriormente citadas para o solo do Parque Estadual das Fontes do Ipiranga (Schoenlein-Crusius et al. 1996).

Rhizopus foi representado por três espécies, $R$. arrhizus, $R$. oligosporus e $R$. oryzae, tendo sido isolado 29 vezes do solo (Tab. 1). Este gênero normalmente está associado ao ambiente terrestre, sendo que as espécies aqui mencionadas são registradas pela primeira vez na Mata Atlântica.

Antunes et al. (1993) isolaram Rhizopus sp. do solo de Paranapiacaba e Schoenlein-Crusius et al. (1996) encontraram Rhizopus nigricans no solo afetado por excrementos de aves no Parque Estadual das Fontes do Ipiranga.

Zygorhynchus, representado pelas espécies Z. japonicus, Z. macrocarpus e Z. moelleri, foi isolado do solo em 17 ocasiões e 16 vezes de folhas de A. triplinervia no ambiente terrestre. As duas primeiras espécies são aqui reportadas pela primeira vez para a Mata Atlântica, enquanto $Z$. moelleri foi verificado anteriormente em folhas submersas de Quercus robur (Schoenlein-Crusius et al. 1990) e também no solo de Paranapiacaba (Antunes et al. 1993).

As espécies Mucor genevensis, Mucor racemosus f. sphaerosporus, Rhizopus oligosporus Z Zygorrhynchus japonicus ocorreram exclusivamente nas folhas colocadas no ambiente terrestre, indicando possível afinidade por substratos em decomposição nesse ambiente. Esta tendência foi confirmada pelos resultados obtidos durante o estudo da sucessão fúngica nas folhas de A. triplinervia colocadas no ambiente terrestre, em que a presença das espécies de Mucorales, nas etapas intermediárias da decomposição estava condicionada à disponibilidade de matéria orgânica e ao teor de nitrogênio total no substrato (Schoenlein-Crusius 1993).

As espécies citadas pela primeira vez para a Mata Atlântica constituem em torno de $50 \%$ do número total de táxons encontrados no presente estudo. Verifica-se, portanto, que a diversidade dos representantes de Mucorales pode se apresentar maior do que indicam os dados publicados até o momento.

\section{Agradecimentos}

Os autores agradecem à Pesquisadora Científica Dra. Sandra Farto Botelho Trufem pelo auxílio na identificação dos táxons e pela revisão do texto. 


\section{Referências bibliográficas}

Antunes, M. F. R.; Ninomiya, A. \& Schoenlein-Crusius, I. H. 1993. Efeitos de queimada sobre a micota de solo na mata atlântica na Reserva Biológica do Alto da Serra de Paranapiacaba, SP. Hoehnea 20(1/ 2): $1-8$

Attili, D. S. 1994. Isolamento, identificação e ecologia de fungos celulolíticos do solo da Estação Ecológica Juréia-Itatins, SP. Rio Claro: Universidade Estadual Paulista. Tese de doutorado. 148p.

Difco Manual. 1972. 9." ed. Michigan: Difco Laboratories. 350p.

Domsch, K. H.; Gams, W. \& Anderson, T. H. 1980. Compendinm of soil fuagi. New York: Academic Press. 860p.

Eggins, H. O. W. \& Pugh, G. J. F. 1962. Isolation of cellulose-decomposing fungi from the soil. Nature 193 (6): 94-95.

Garlipp, A. B. 1995. Isolamento e identificação de fungos filamentosos do solo do Banhado Grande na Estação Ecológica de Juréia-Itatins. Rio Claro: Universidade Estadual Paulista. Dissertação de mestrado. 94p.

Hawksworth, P. M.; Kirk, P. M.; Sutton, B. C. \& Pegler, D. N. 1995. Ainsworth \& Bisby's Dictionary of the fungi. 8." ed. International Mycological Institute. Cambridge, University Press. 616p.

Hesseltine, C. W. \& Ellis, J. J. 1973. Mucorales. In: G. C. Ainsworth; F. K. Sparrow \& A. S. Sussman (eds), The fungi: an advanced treatise. New York: Academic Press. Vol. 4B, p. 187-217.

Lacaz, C. S.; Minami, P. S. \& Purchio, A. 1970. O grande mundo dos fungos. São Paulo: EDUSP, ed. Polígono. 255p

Maia, L. C. 1983. Sucessão de fungos em folhedo de Floresta Tropical Úmida. Recife: Universidade Federal de Pernambuco, Editora Universitária, 83p.

Pugh, G. J. F.; Buckey, N. G. \& Mulder, J. 1972. The role of phylloplane fungi in the early colonization of leaves. Symposium Biological Hungarica 11: 329-333.

Schipper, M. A. A. 1973. A study on variability in Mucor hiemalis and related species. Studies in Mycology 4: $1-40$.

Schipper, M. A. A. 1976. On Mucor circinelloides, Mucor racemosus and related species. Studies in Mycology 11: $1-35$.

Schoenlein-Crusius I. H. 1993. Sucessão fúngica em folhas de Alchornea triplinervia (Spreng.) M. Arg. em ambientes aquático e terrestre, na mata atlântica, Reserva Biológica do Alto da Serra de Paranapincaba, Santo André, SP. Rio Claro: Universidade Estadual Paulista. Tese de doutorado. $373 \mathrm{p}$.

Schoenlein-Crusius, I. H. \& Milanez, A. I. 1989. Sucessão fúngica em folhas de Ficus microcarpa L.f submersas no lago frontal situado no Parque Estadual das Fontes do Ipiranga, São Paulo, SP. Revista de Microbiologia 20(1): 95- 101.

Schoenlein-Crusius, I. H. \& Tauk, S. M. 1991. Fungal succession on Ocotea pulchella (Nees) Mez leaves in decomposition on "cerrado" soil treated with vinasse. Revista de Microbiologia 22(2): 179-183.

Schoenlein-Crusius, I. H.; Pires-Zottarelli, C. L. A. \& Milanez, A. I. 1990. Sucessāo fúngica em folhas de Quercus robur L. (carvalho) submersas em um lago situado no município de Itapecerica da Serra, SP Revista de Microbiologia 21(1): 61-67.

Schoenlein-Crusius, I. H.; Pires-Zottarelli, C. L. A. \& Okino, L. K. 1994. I Catálogo da Coleção de Culturas de Fungos. São Paulo: Instituto de Botânica. 49p.

Schoenlein-Crusius, I. H.; Trufem, S. F. B.; Malatinsky, S. M. M.; Ninomiya, A. \& Antunes, M. F. R. 1996. Mucorales (Zygomycotina) from soil affected by excrement of birds in the "Parque Estadual das Fontes do Ipiranga", Sao Paulo, Brazil. Revista Brasileira de Botânica 19(1): 7-10.

Trufem, S. F. B. 1978. Mucorales (Zygomycetes) do Estado de São Paulo. São Paulo: Escola Paulista de Medicina. Dissertação de mestrado. 178p.

Trufem, S. F. B. 1981a. Mucorales do Estado de São Paulo. 1. Mucor Micheli. Rickia 9: 81-91

Trufem, S. F. B. 1981b. Mucorales do Estado de São Paulo. 2. Gêneros Absidia van Tieghem, Gongronella 
Ribaldi e Rhizopus Ehrenberg. Rickia 9: 99- 106.

Trufem, S. F. B. 1981c. Mucorales do Estado de São Paulo. 3. Gêneros Circinella van Tieghem \& Le Monnier e Cunninghamella Matruchot. Rickia 9: 113-120

Trufem, S. F. B. 1984. Mucorales do Estado de São Paulo: 4. Espécies coprófilas. Rickia 11: 53-64.

Trufem, S. F. B. \& Viriato, A. 1985. Mucorales do Estado de São Paulo: 6. Mucoraceae coprófilas. Rickia 12: 113-123.

Viriato, A. 1996. Diversidade e aspectos ecológicos de Mucorales (Zygomycetes) de solo e de fezes de herbívoros, no Parque Estadual das Fontes do Ipiranga, São Paulo, Brasil. São Paulo: Universidade de Guarulhos. 162p

Viriato, A. \& Trufem, S.F.B. 1985. Mucorales do Estado de São Paulo: 5. Pilobolaceae. Rickia 12: 77-88.

Warcup, J. H. 1950. The soil plate method for isolations of fungi from soil. Nature 166(2): 117-118.

Zycha, H.; Siepmann, R. \& Linnemann, G. 1969. Mucorales einer Beschreibung aller Gatungen und Arten dieser Pilzgruppe. Leipzig: Von Cramer. 355p. 